\title{
KONGRESSKALENDER
}

\section{Mai 2012}

18.05.2012, Dresden

17. Dresdner Frühjahrstagung für

Forensische Psychiatrie

Die forensische Relevanz der geistigen

Behinderung - Diagnostik, Begutachtung,

Behandlung.

Dr. Sutarski, Dr. Lammel

Information: www.forensik-dresden.de

19.05.2011, Wien, Allgemeines Krankenhaus

Wien

6. Wiener Frühjahrstagung für

Forensische Psychiatrie

Schizophrenie und Gefährlichkeit

Prof. Dr. Th. Stompe

Information: Yasmin Haunold, office@iec-

management.at

\section{Juni 2012}

08.06.2012, Berlin-Dahlem

16. Berliner Junitagung für Forensische Psychiatrie

Anatomie und Rahmenstrukturen

von Gewalt - Junge Gewalttäter. Alte

Gewalttäter.

Institut für Forensische Psychiatrie der

Charité

Information: www.forensik-berlin.de

(Veranstaltungen)

11.-13.06.2012, Stockholm, Schweden

Stockholm Symposium

Stockholm City Conference Center

Information: www.criminologyprize.com/

extra/pod/

\section{Juli 2012}

05.-06.07.2012, Regensburg

8th International Summer Conference

Research in Forensic Psychiatry

Prof. Dr. Osterheider, Universität Regensburg

Information: http://www.forensik-regensburg.

de

\section{August 2012}

20.-24.08.2012, Den Haag, Niederlande

6th European Academy of Forensic Science

Conference

EAFS

Information: http://www.eafs2012.eu

\section{September 2012}

05.-08.09.2012, Berlin, Charité Campus

Virchow Klinikum

12th IATSO Conference

International Association for the Treatment for Sexual Offenders

Information: www.iatso.org

03.-07.09.2012, Königslutter

Curriculum Forensische Psychiatrie

und Psychologie

Die psychiatrisch-psychologische

Begutachtung im Strafverfahren, Teil 2

Prof. Dr. N. Konrad, Dr. U. Baltzer,

Dr. Ch. Herbig

Information: www.psychiatrie-akademie.de

07.-09.09.2012, Königslutter

Curriculum Forensische Psychiatrie

und Psychologie

Die psychiatrisch-psychologische

Begutachtung im Strafverfahren, Teil 3

Prof. Dr. N. Konrad, Dr. U. Baltzer,

Dr. Ch. Herbig

Information: www.psychiatrie-akademie.de

10.-12.09.2012, Potsdam-Griebnitzsee

16. Fallseminar für Forensische

Psychiatrie

Therapieverlaufsbeurteilung;

Gutachtenmängel

Prof. Dr. H.-L. Kröber

Institut für Forensische Psychiatrie der

Charité

Information: www.forensik-berlin.de

12.-15.09.2012, Bilbao, Spanien

12th Annual ESC Conference

European Association of Criminology

Information: http://www.esc-eurocrim.org/

26.09.2012, Hemer

6. Dortmund-Hemeraner Fachgespräch zur Forensischen Psychiatrie

Warum verhalten wir uns prosozial und sind keine Psychopathen?

Hans-Prinzhorn-Klinik Hemer

Information:wrk-sekretariat@wkp-lwl.org

\section{Oktober 2012}

11.-13.10.2012, München

27. Herbsttagung für Forensische

Psychiatrie

Prof. Dr. N. Nedopil

Abteilung für Forensische Psychiatrie der

Universität München

Information: www.forensik-muenchen.de
25.-28.10.2012, Montreal QC, Canada

American Academy of Psychiatry

and the Law

Annual Meeting (with APA)

Le Centre Sheraton

Information: www.AAPL.org

\section{November 2012}

14.-17.11.2012 Chicago IL, USA

American Society of Criminology

Annual Meeting

Palmer House Hilton

Information: http://www.asc41.com/

annualmeeting.htm

Ein internationaler kriminologischer Kongresskalender findet sich unter http://www.asc41.com/caw.html

Hinweise für den Kongresskalender bitte an: hans-ludwig.kroeber@charite.de 